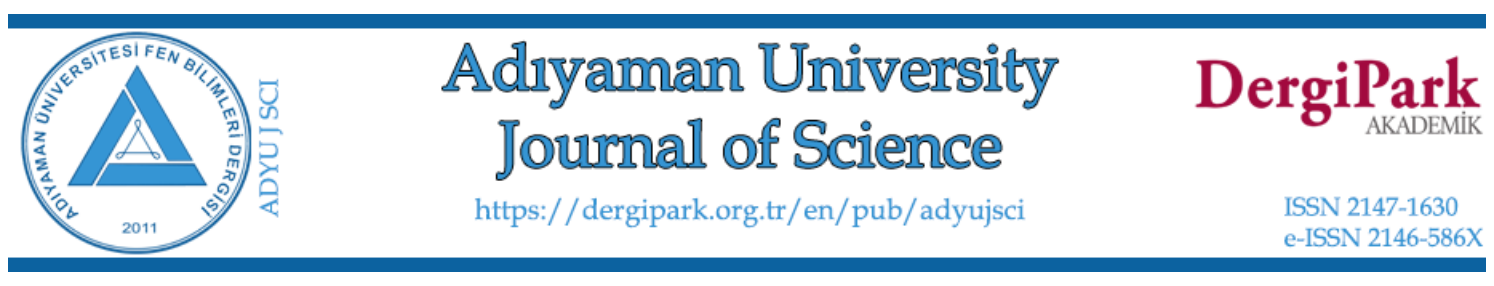

\title{
Optical Solutions of the Kundu-Eckhaus Equation via Two Different Methods
}

\author{
Melike KAPLAN ${ }^{1, *}$ \\ ${ }^{1}$ Kastamonu University, Faculty of Art and Science, Department of Mathematics, 37150, Kastamonu, \\ Turkey \\ mkaplan@kastamonu.edu.tr, ORCID: 0000-0001-5700-9127
}

Received: 10.12 .2020

Accepted: 14.05 .2021

Published: 30.06 .2021

\begin{abstract}
This work is devoted to obtaining new optical solutions to the Kundu-Eckhaus (KE) equation which is believed to play a crucial part in the area of nonlinear optics. Two different methods, the $\exp (-\varphi(\varepsilon))$ method with the exponential rational function approach have been utilized. Both methods are efficient in finding the analytical solutions of many nonlinear partial differential equations and fractional differential equations. Results obtained in this research are dissimilar to the ones in the literature and the solutions are controlled by relocating them back to the primary equation. Finally, it can be stated that optical solutions have a promising future.
\end{abstract}

Keywords: Nonlinear equation; Symbolic computation; Optical solutions.

\section{Kundu-Eckhaus Denkleminin İki Farklı Yöntemle Optik Çözümleri}

\section{Öz}

$\mathrm{Bu}$ çalışmada, lineer olmayan optik alanında önemli bir yere sahip olan Kundu-Eckhaus (KE) denkleminin optik çözümlerinin elde edilmesine yer verilmiştir. $\exp (-\varphi(\varepsilon))$ yöntemi ve üstel rasyonel fonksiyon yöntemi ilgili denkleme uygulanmıştır. Bahsedilen her iki yöntem de lineer olmayan kısmi diferensiyel denklemler ve kesir mertebeden diferensiyel denklemlerin tam çözümlerinin elde edilmesinde oldukça etkili olduğu bilinen yöntemlerdir. Bu çalışmada elde 
edilen sonuçlar, literatürde daha önce var olanlardan farklıdır. Elde edilen çözümler, Maple yardımıyla yerine konularak kontrol edilmiştir. Sonuç olarak, optik çözümlerin literatürde önemli bir geleceğinin olduğunu belirtmeliyiz.

Anahtar Kelimeler: Lineer olmayan denklem; Sembolik hesaplama; Optik çözümler.

\section{Introduction}

A lot of real-world problems in the vast areas of engineering and science are modeled by nonlinear evolution equations (NLEEs). Looking for the exact solutions of NLEEs is quite crucial for one to understand the phenomena described by the NLEEs. The analytical expressions of NLEEs were researched using various powerful techniques. Some of these methods are AutoBacklund transformations[1], modified simple equation method [2], transformed rational function method [3], trial method [4], the sine-Gordon expansion method [5], the (G'/G)- expansion method [6], (G'/G,1/G)-expansion method [7], auxiliary equation method [8, 9], exp-function method [10], F-expansion method [11], sine-cosine method [12], ansatz method [13], sub equation method [14], exponential rational function method [15], Lie group analysis [16], Hirota bilinear method [17], Backlund transformation method [18], Wronskian technique [19], homogeneous balance method [20], inverse scattering method [21], and so on [22]. Regarding the domain of research in photonics sciences, optical solitons is considered among the most rapidly emerging fields. Recently, this technique is widely used by scientists [23, 24, 25].

The $\exp (-\varphi(\varepsilon))$ method is a new and useful method that gives many solutions to NLEEs. The supremacy of the suggested technique toward the $\left(\mathrm{G}^{\prime} / \mathrm{G}\right)$-expansion scheme requires to give fresh travelling wave solutions while using arbitrary additional parameters [26, 27]. The other technique utilized in the paper, the exponential function method, is a straight method.

The style implemented in this article can be described as follows: In the Part of Materials and Methods, the primary stages of methods are introduced. In Section III, the implementation of the techniques to the Kundu-Eckhaus is given. Finally, the conclusion of the paper is provided.

\section{Materials and Methods}

A general NLEE of the formula will be taken into consideration:

$$
P\left(u, u_{x}, u_{t}, u_{x x}, u_{x t}, u_{t t}, \ldots\right)=0 \text {, }
$$

The polynomial and derivates of $u=u(x, t)$ are represented by $P$, where the nonlinear terms and the highest order derivatives are comprised. In a suitable manner of the following travelling wave transformation 


$$
\xi=x-c t, u(x, t)=U(\xi),
$$

where c represents the velocity of the wave, Eqn. (1) is reduced thereby forming an ordinary differential equation (ODE) in the form

$$
Q\left(U, U^{\prime}, U^{\prime \prime}, U^{\prime \prime \prime}, \ldots\right)=0 \text {. }
$$

It is noted that, in Eqn. (2) the differentiation of $U$ with respect to $\xi$ is represented by prime. All the terms in Eqn. (2) will be integrated.

\subsection{The $\exp (-\varphi(\xi))$ method}

Conforming to this technique [27], the desired solution for the reduced equation is formed by a polynomial in $\exp (-\varphi(\xi))$ as

$$
\mathrm{U}(\xi)=\sum_{\mathrm{n}=0}^{\mathrm{m}} a_{n}(\exp (-\varphi(\xi)))^{\mathrm{n}},
$$

where $a_{n},\left(a_{m} \neq 0\right)$ are constants which will be found afterwards and $\varphi(\xi)$ is the solution of the following ODE below

$$
\varphi^{l}(\xi)=\exp (-\varphi(\xi))+\mu \exp (\varphi(\xi))+\lambda .
$$

The supplementary equation Eqn. (3) possesses distinct solutions below:

Case 1: When $\lambda^{2}-4 \mu>0$ and $\mu \neq 0$, the hyperbolic function solutions,

$$
\varphi_{1}(\xi)=\ln \left(\frac{-\sqrt{\lambda^{2}-4 \mu} \tanh \left(\sqrt{\frac{\lambda^{2}-4 \mu}{2}}(\xi+C)\right)-\lambda}{2 \mu}\right) .
$$

Case 2: When $\lambda^{2}-4 \mu<0$ and $\mu \neq 0$, trigonometric function solutions,

$$
\varphi_{2}(\xi)=\ln \left(\frac{\left.\sqrt{4 \mu-\lambda^{2}} \tan h \sqrt{\frac{4 \mu-\lambda^{2}}{2}}(\xi+C)\right)-\lambda}{2 \mu}\right) .
$$

Case 3: When $\lambda^{2}-4 \mu>0, \mu \neq 0$ and $\lambda \neq 0$, hyperbolic function solutions,

$$
\varphi_{3}(\xi)=-\ln \left(\frac{\lambda}{\cosh (\lambda(\xi+C))+\sinh (\lambda(\xi+C))-1}\right) .
$$

Case 4: When $\lambda^{2}-4 \mu=0, \mu \neq 0$ and $\lambda \neq 0$, rational function solutions,

$$
\varphi_{4}(\xi)=\ln \left(-\frac{2(\lambda+(\xi+C)+2)}{\lambda^{2}(\xi+C)}\right)
$$


Case 5: When $\lambda^{2}-4 \mu=0, \mu=0$ and $\lambda=0$,

$$
\varphi_{5}(\xi)=\ln (\xi+C) \text {. }
$$

\subsection{The exponential rational function method}

In agreement with this technique, Eqn. (2)'s solitary wave solution is assumed as [21, 22]:

$$
\mathrm{U}(\xi)=\sum_{\mathrm{n}=0}^{\mathrm{N}} \frac{\mathrm{a}_{\mathrm{n}}}{\left(1+\exp (\xi)^{n}\right.}
$$

where $a_{n}\left(a_{N} \neq 0\right)$ are constants which will be determined afterwards. It can be noted that congruently $\mathrm{N}$ is a balancing number. Here the same logic steps in and, on subrogating Eqn. (9) in Eqn. (2), then gathering all the terms in the similar order of $\exp (i \xi),(i=0,1,2, \ldots)$, the left-hand side of Eqn. (2) is converted into a new polynomial in $\exp (i \xi)$. Later on, one can equate every coefficient of the obtained polynomial to zero in order to solve the system. Consequently, the solutions of the system can be calculated using the assistance of the Maple that gives the desired solitary wave solutions of Eqn. (1).

\section{Results and Discussion}

\subsection{Mathematical analysis}

Kundu-Eckhaus (KE) equation, founded by Kundu [28] and Eckhaus [29], is chosen to implement how the methods work. This equation supplies a different model for the solitons transmission along optical fibers. This equation, within the nonlinear Schrödinger class, is a fundamental model which is modelling optical soliton promulgation in Kerr media. The magnitude of the incident light field to generate ultrashort (femtosecond) optical pulses in optic fiber communications systems must be adjusted [30].

$$
i \frac{\partial^{\alpha} q(x, t)}{\partial t^{\alpha}}+a \frac{\partial^{2} q(x, t)}{\partial x^{2}}+b|q(x, t)|^{4} q(x, t)+c \frac{\partial\left(|q(x, t)|^{2}\right)}{\partial x} q(x, t)=0 .
$$

Here complex-valued wave profile represents by $q(x, t)$. Eqn. (10)'s first term involves the temporal evolution of the nonlinear wave, whereas the real-valued constants $c, b$ and $a$ denote, nonlinear effect, quintic nonlinearity, group velocity dispersion and respectively.

Analysis of Eqn. (10) gives:

$$
q(x, t)=\sqrt{v(s)} e^{i \Phi(x, t)},
$$

where $v(s)$ denotes the form of the pulse and

$$
s=k\left(x+2 a \kappa \frac{t^{\alpha}}{\alpha}\right),
$$


and the phase component is denoted as shown below.

$$
\Phi(x, t)=-\kappa x+\omega\left(\left(t^{\alpha}\right) / \alpha\right)+\theta_{0}
$$

where $\omega=$ wave number of the soliton, $\kappa=$ soliton frequency, and $\theta_{0}=$ phase constant.

Then by subrogating Eqn. (11) in Eqn. (10) and dividing them into imaginary and real parts the following equation is obtained.

$$
2 a k^{2} v v^{\prime \prime}+4 c k v^{2} v^{\prime}-a k^{2}\left(v^{\prime}\right)^{2}-4\left(\omega+a \kappa^{2}\right) v^{2}+4 b v^{4}=0 .
$$

According to homogenous balance principle, balancing $v v^{\prime \prime}$ with $v^{4}$ gives $N=1$.

\subsection{The $\exp (-\varphi(\xi))$ method}

In accordance with idea of the adopted method gives the solution of Eqn. (12) below.

$$
v(\xi)=a_{0}+a_{1} \exp (-\varphi(\xi)) .
$$

The equation system consisting of $a, b, c, k, a_{0}, a_{1}, \lambda, \mu, \omega, \kappa$ could be found by substituting Eqn. (13) into Eqn. (12), gathering the coefficient of every term of $\exp (-\varphi(\xi))^{\mathrm{n}}$ and equating all coefficients to zero.

$$
\begin{aligned}
\exp (4 \xi): & -a k^{2} a_{1}{ }^{2} \mu^{2}-4 c k a_{1} a_{0}{ }^{2} \mu+4 b a_{0}{ }^{4}-4 \omega a_{0}{ }^{2} \\
& -4 a \kappa^{2} a_{0}{ }^{2}+2 a k^{2} a_{0} a_{1} \mu \lambda=0, \\
\exp (3 \xi): & -8 \omega a_{0} a_{1}+16 b a_{0}{ }^{3} a_{1}+2 a k^{2} a_{0} a_{1} \lambda^{2}-8 a \kappa^{2} a_{0} a_{1} \\
& -4 c k a_{1} a_{0}{ }^{2} \lambda-8 c k a_{1}{ }^{2} a_{0} \mu+4 a k^{2} a_{0} a_{1} \mu=0, \\
\exp (2 \xi): & -4 c k a_{1} a_{0}{ }^{2}+6 a k^{2} a_{0} a_{1} \lambda-4 c k a_{1}{ }^{3} \mu-8 c k a_{1}{ }^{2} a_{0} \lambda \\
& -4 a \kappa^{2} a_{1}{ }^{2}-4 \omega a_{1}{ }^{2}+24 b a_{0}{ }^{2} a_{1}{ }^{2}+a a_{0}{ }^{2} a_{1}{ }^{2} \lambda^{2}+2 a k^{2} a_{1}{ }^{2} \mu=0, \\
\exp (1 \xi): & 4 a k^{2} a_{0} a_{1}+16 b a_{0} a_{1}{ }^{3}-8 c k a_{1}{ }^{2} a_{0}-4 c k a_{1}{ }^{3} \lambda+4 a k^{2} a_{1}{ }^{2} \lambda=0, \\
\exp (0 \xi): & 3 a k^{2} a_{1}{ }^{2}-4 c k a_{1}{ }^{3}+4 b a_{1}{ }^{4}=0 .
\end{aligned}
$$

Utilisation of software programs gives the solution as follows:

$$
a_{0}=\frac{\left(\frac{\lambda}{2}+\frac{\sqrt{\lambda^{2}-4 \mu}}{2}\right) c k}{4 b}, \quad a_{1}=\frac{c k}{4 b}, \quad a=\frac{c^{2}}{4 b}, \quad \omega=-\frac{\left(4 \kappa^{2}-k^{2} \lambda^{2}+4 k^{2} \mu\right) c^{2}}{16 b} .
$$

These obtained values, the algorithm of the method and its auxiliary equations, gives the various optical solutions for KE equation: 
Case 1: $\lambda^{2}-4 \mu>0$ and $\mu \neq 0$, according to Eqn. (4),

The hyperbolic function solutions:

$q_{1}(x, t)=\sqrt{\frac{\left(\frac{\lambda}{2}+\frac{\sqrt{\lambda^{2}-4 \mu}}{2}\right) c k}{4 b}-\frac{2 c k \mu}{4 b \sqrt{\lambda^{2}-4 \mu} \tanh \left(\frac{\sqrt{\lambda^{2}-4 \mu}}{2}\left(k x+2 a k \kappa \frac{t^{\alpha}}{\alpha}\right)\right)+4 b \lambda}} \times \exp \left(i\left(-\kappa x+\omega \frac{t^{\alpha}}{\alpha}+\theta_{0}\right)\right)$.

Case 2: $\lambda^{2}-4 \mu<0$ and $\mu \neq 0$, according to Eqn. (5),

The trigonometric function solutions:

$q_{2}(x, t)=\sqrt{\frac{\left(\frac{\lambda}{2}+\frac{\sqrt{\lambda^{2}-4 \mu}}{2}\right) c k}{4 b}+\frac{2 c k \mu}{4 b \sqrt{4 \mu-\lambda^{2}} \tan \left(\frac{\sqrt{4 \mu-\lambda^{2}}}{2}\left(k x+2 a k \kappa \frac{t^{\alpha}}{\alpha}\right)\right)-\lambda}} \times \exp \left(i\left(-\kappa x+\omega \frac{t^{\alpha}}{\alpha}+\theta_{0}\right)\right)$.

Case 3: $\lambda^{2}-4 \mu>0, \mu=0$ and $\lambda \neq 0$, according to Eqn. (6),

The hyperbolic function solutions:

$$
\begin{aligned}
q_{3}(x, t)= & \sqrt{\frac{\left(\frac{\lambda}{2}+\frac{\sqrt{\lambda^{2}-4 \mu}}{2}\right) c k}{4 b}+\frac{c k \lambda}{4 b \cosh \left(\lambda\left(k x+2 a k \kappa \frac{t^{\alpha}}{\alpha}+C\right)\right)+4 b \sinh \left(\lambda\left(k x+2 a k \kappa \frac{t^{\alpha}}{\alpha}+C\right)\right)-4 b}} \\
& \times \exp \left(i\left(-\kappa x+\omega \frac{t^{\alpha}}{\alpha}+\theta_{0}\right)\right) .
\end{aligned}
$$

Case 4: When $\lambda^{2}-4 \mu=0, \mu \neq 0$ and $\lambda \neq 0$, according to Eqn.(7),

The rational function solutions:

$q_{4}(x, t)=\sqrt{\frac{\left(\frac{\lambda}{2}+\frac{\sqrt{\lambda^{2}-4 \mu}}{2}\right) c k}{4 b}-\frac{\lambda^{2} c k\left(k x+2 a k \kappa \frac{t^{\alpha}}{\alpha}+C\right)}{8 b \lambda k x+16 b \lambda a k \kappa \frac{t^{\alpha}}{\alpha}+8 b C+16 b}} \times \exp \left(i\left(-\kappa x+\omega \frac{t^{\alpha}}{\alpha}+\theta_{0}\right)\right)$.

Case 5: $\lambda^{2}-4 \mu=0, \mu=0$ and $\lambda=0$, according to Eqn.(8),

$q_{5}(x, t)=\sqrt{\frac{c k}{4 b k x+8 b a k \kappa \frac{t^{\alpha}}{\alpha}+4 b C}} \times \exp \left(i\left(-\kappa x+\omega \frac{t^{\alpha}}{\alpha}+\theta_{0}\right)\right)$.

\subsection{The exponential rational function method}

Optical solutions of the reduced form of KE equation which is Eqn. (12) can be assumed as follows: 


$$
v(\xi)=a_{0}+\frac{a_{1}}{1+\exp (\xi)}
$$

a polynomial of $\exp (i \xi)(i=0,1, \ldots 4)$ can be obtained. Then by adjusting the powers of the obtained polynomial to zero, one can find the equation system below.

$$
\begin{aligned}
\exp (4 \xi): & -4 a \kappa^{2} a_{0}{ }^{2}-4 \omega a_{0}{ }^{2}+4 b a_{0}{ }^{4}=0 \\
\exp (3 \xi): & 2 a k^{2} a_{0} a_{1}+16 b a_{0}{ }^{3} a_{1}-16 a \kappa^{2} a_{0}{ }^{2}-16 \omega a_{0}{ }^{2}-8 \omega a_{0} a_{1} \\
& -8 a \kappa^{2} a_{0} a_{1}-4 c k a_{1} a_{0}{ }^{2}+16 b a_{0}{ }^{4}=0, \\
\exp (2 \xi): & -24 a \kappa^{2} a_{0} a_{1}-24 a \kappa^{2} a_{0}{ }^{2}+24 b a_{0}{ }^{2} a_{1}{ }^{2}+48 b a_{0}{ }^{3} a_{1}-4 a \kappa^{2} a_{1}{ }^{2}-4 \omega a_{1}{ }^{2} \\
& -24 \omega a_{0}{ }^{2}-24 \omega a_{0} a_{1}+24 b a_{0}{ }^{4}+a k^{2} a_{1}{ }^{2}-8 c k a_{1}{ }^{2} a_{0}-8 c k a_{1} a_{0}{ }^{2}=0, \\
\exp (1 \xi): & -2 a k^{2} a_{0} a_{1}-4 c k a_{1} a_{0}{ }^{2}-16 a \kappa^{2} a_{0}{ }^{2}-2 a k^{2} a_{1}{ }^{2}-4 c k a_{1}{ }^{3} \\
& -24 a \kappa^{2} a_{0} a_{1}-8 a \kappa^{2} a_{1}{ }^{2}+48 b a_{0}{ }^{2} a_{1}{ }^{2}+48 b a_{0}{ }^{3} a_{1} \\
+ & 16 b a_{0} a_{1}{ }^{3}-8 c k a_{1}{ }^{2} a_{0}-16 \omega a_{0}{ }^{2}-24 \omega a_{0} a_{1}+16 b a_{0}{ }^{4}-8 \omega a_{1}{ }^{2}=0, \\
\exp (0 \xi): & -4 \omega a_{0}{ }^{2}+4 b a_{0}{ }^{4}-4 \omega a_{1}{ }^{2}+4 b a_{1}{ }^{4}-4 a \kappa^{2} a_{0}{ }^{2}-8 \omega a_{0} a_{1}-4 a \kappa^{2} a_{1}{ }^{2} \\
& +16 b a_{0}{ }^{3} a_{1}+24 b a_{0}{ }^{2} a_{1}{ }^{2}+16 b a_{0} a_{1}{ }^{3}-8 a \kappa^{2} a_{0} a_{1}=0 .
\end{aligned}
$$

From the solutions of the algebraic equations using Maple, two different cases can be verified as follows:

\section{Case 1:}

$$
a_{0}=0, \quad a_{1}=-\frac{c k}{4 b}, a=\frac{c^{2}}{4 b}, \quad \omega=\frac{c^{2}\left(-4 \kappa^{2}+k^{2}\right)}{16 b}
$$

Then, on substituting these results into Eqn. (14) in order to obtain the optical solutions of KE equation gives

$$
q_{6}(x, t)=\sqrt{-\frac{c k}{4 b\left(1+\exp \left(k\left(x+2 a \kappa \frac{t^{\alpha}}{\alpha}\right)\right)\right)}} \exp \left(i\left(-\kappa x+\omega \frac{t^{\alpha}}{\alpha}+\theta_{0}\right)\right) .
$$

\section{Case 2:}

$$
a_{0}=\frac{c k}{4 b}, a_{1}=-\frac{c k}{4 b}, a_{2}=\frac{c^{2}}{4 b}, \omega=\frac{c^{2}\left(-4 \kappa^{2}+k^{2}\right)}{16 b}
$$

Then, on substituting these results into Eqn. (14) in order to obtain the optical solutions of KE equation gives

$$
q_{7}(x, t)=\sqrt{\frac{c k}{4 b}-\frac{c k}{4 b\left(1+\exp \left(k\left(x+2 a \kappa \frac{t^{\alpha}}{\alpha}\right)\right)\right)}} \exp \left(i\left(-\kappa x+\omega \frac{t^{\alpha}}{\alpha}+\theta_{0}\right)\right) .
$$


The KE equation was considered successful according to the $\exp (-\varphi(\xi))$ and the exponential rational methods. As an upshot, several wave solutions have been previously acquired. Also, one must note that the accuracy of the acquired optical solutions was checked by subrogating every solution to KE equation. Since the $\exp (-\varphi(\xi))$ method gives various types of solutions, it can be applied to additional models that will be considered in near future. Since the exponential rational function method is direct and effective, it is more simple to implement.

\section{Conclusion}

The results in this paper were dissimilar to the existing ones in the literature, and the results/they were checked thanks to Maple by resubstituting the solutions into the Kundu-Eckhaus equation. Finally, it could be stated that optical solutions have a promising future. Since both methods are applicable for many nonlinear partial differential equations, further applications are implementable for future studies. Also, different types of exact solutions can be founded for the dealt equation by using different methods.

\section{References}

[1] Kaplan M., Ozer, M.N., Auto-Bäcklund transformations and solitary wave solutions for the nonlinear evolution equation, Optical and Quantum Electronics, 50(1), 33, 2018.

[2] Akter, J., Akbar, M.A., Exact solutions to the Benney-Luke equation and the Phi-4 equations by using modified simple equation method, Results in Physics, 5, 125-130, 2015.

[3] Ma, W.X., Lee, J.H., A transformed rational function method and exact solutions to the 3+1 dimensional Jimbo-Miwa equation, Chaos, Solitons and Fractals, 42, 1356-1363, 2009.

[4] Mirzazadeh, M., Arnous, A.H., Mahmood, M.F. Zerrad, E. Biswas, A., Soliton solutions to resonant nonlinear Schrödinger's equation with time-dependent coefficients by trial solution approach, Nonlinear Dynamics, 81, 277-282, 2015.

[5] Bulut, H., Akturk, T., Gurefe, Y., An application of the new function method to the generalized double sinh-Gordon equation, AIP Conference Proceedings 1648, 370014, 2015.

[6] Islam, M.S., Khan, K., Akbar, M. A., An analytical method for finding exact solutions of modified Korteweg-de Vries equation, Results in Physics, 5, 131-135, 2015.

[7] Inan, I.E., Ugurlu, Y., Inc, M., New Applications of the $\left(G^{\prime} / G, 1 / G\right)$-Expansion Method, Acta Physica Polonica A, 128, 245-251, 2015.

[8] Abdou, M.A., A generalized auxiliary equation method and its applications, Nonlinear Dynamics, 52, 95-102, 2008.

[9] Adem, A.R., Khalique, C.M., Conserved quantities and solutions of a $(2+1)$ dimensional Haragus-Courcelle-Il'ichev model, Computers and Mathematics with Applications, 71, 1129-1136, 2016.

[10] He, J.H., Abdou, M.A., New periodic solutions for nonlinear evolution equations using Exp-function method, Chaos, Solitons and Fractals, 34, 1421-1429, 2007.

[11] Abdou, M.A., Further improved F-expansion and new exact solutions for non- linear evolution equations, Nonlinear Dynamics, 52, 277-288, 2008. 
[12] Mirzazadeh, M., Eslami, M., Zerrad, E., Mahmood, M.F., Biswas, A., Belic, M., Optical solitons in nonlinear directional couplers by sine-cosine function method and Bernoulli's equation approach, Nonlinear Dynamics, 81, 1933-1949, 2015.

[13] Younis, M., Ali, S. , Mahmood, S.A., Solitons for compound KdV-Burgers equation with variable coefficients and power law nonlinearity, Nonlinear Dynamics, 81, 1191-1196, 2015.

[14] Durur, H., Kurt, A., Tasbozan, O., New Travelling Wave Solutions for KdV6 Equation Using Sub Equation Method, Applied Mathematics and Nonlinear Sciences, 5(1), 455-460, 2020.

[15] Yusufoğlu, E., New solitonary soltions for the MBBM equatios using Exp-function Method, Physic Letters A, 372, 442-446, 2008.

[16] Biswas, A., Khalique, C.M., Stationary solutions for nonlinear dispersive Schrödinger's equation, Nonlinear Dynamics, 63, 623-626, 2011.

[17] Wazwaz, A.M., Multiple-soliton solutions for the Boussinesq equation, Applied Mathematics and Computation, 192, 479-486, 2007.

[18] Lü, X., Tian, B., Zhang, H.Q., Xu, T., Li, H., Generalized (2+1)-dimensional Gardner model: bilinear equations, Bäcklund transformation, Lax representation and interaction mechanisms, Nonlinear Dynamics, 67, 2279-2290, 2012.

[19] Ma, W.X., Abdeljabbar, A., Asaad, M.G., Wronskian and Grammian solutions to a $(3+1)$-dimensional generalized KP equation, Applied Mathematics and Computation, 217, 10016-10023, 2011.

[20] Wang, M.L., Solitary wave solutions for variant Boussinesq equations, Physics Letters A, 199, 169-172, 1995.

[21] Ablowitz, M.J., Segur, H., Solitons and Inverse Scattering Transformation, SIAM, Philadelphia, 1981.

[22] Thabet, H., Kendre, S., Peters, J., Kaplan, M., Solitary wave solutions and traveling wave solutions for systems of time-fractional nonlinear wave equations via an analytical approach, Computational and Applied Mathematics, 39, 2020, 144.

[23] Zayed, E.M.E., Alngar, M.E.M., Al-Nowehy, A.G., On solving the nonlinear Schrödinger Equation with an anti-cubic nonlinearity in presence of Hamiltonian perturbation terms, Optik - International Journal for Light and Electron Optics, 178, 488-508, 2019.

[24] Biswas, A., Jawad, A.J.M. and Zhou, Q., Resonant optical solitons with anti-cubic nonlinearity, Optik, 157, 525-531, 2018.

[25] Biswas, A., Optical soliton perturbation with Radhakrishnan-Kundu-Lakshmanan equation by traveling wave hypothesis, Optik, 171, 217-220, 2018.

[26] Kaplan, M., Application of two reliable methods for solving a nonlinear conformable time-fractional equation, Optical and Quantum Electronics, 49, 312, 2017.

[27] Roshid, H.O., Kabir, M.R., Bhowmik, R.C., Datta, B.K., Investigation of Solitary wave Solutions for Vakhnenko-Parkes equation via exp-function and $\exp (-\varphi(\xi))$-expansion method. SpringerPlus, 3, 692, 2014.

[28] Kundu, A., Landau-Lifshitz and higher-order nonlinear systems gauge generated from nonlinear Schrödinger type equations, Journal of Mathematical Physics, 25, 3433-3438, 1984.

[29] Eckhaus, W., The long-time behaviour for perturbed wave-equations and related problems, Preprint no. 404, Department of Mathematics, University of Utrecht, 1985.

[30] Mirzazadeh, M., Yıldırım, Y., Yas, E., Triki, H., Zhoud, Q., Moshokoa, S.P., Ullah, M.Z., Seadawy, A.R., Biswas, A., Belic, M., Optical solitons and conservation law of Kundu- 
Kaplan (2021) ADYU J SCI, 11(1), 126-135

Eckhaus equation, Optik, 154, 551-557, 2018. 